\title{
Identifying inmates that will actually die by suicide
}

\author{
Vladimir Carli
}

Karolinska Instituet, National Centre for Suicide Research and Prevention of Mental Ill-Health (NASP), Granits Vag 4 Stockholm 17177,

Sweden; vladimir.carli@ki.se

\section{WHAT IS ALREADY KNOWN ON THIS TOPIC?}

Suicide rates among prisoners are higher than in the general population. ${ }^{1}$ It has also been shown that specific risk factors, such as violent behaviour in prison, psychiatric diagnosis, substance abuse and previous suicide attempts are associated with self-harm in prison. ${ }^{2}$ Fifty per cent of prisoners who die by suicide have a history of self-harm ${ }^{3}$ and longitudinal studies are needed to understand which prisoners with self-harm are more likely to complete suicide.

\section{WHAT THIS PAPER ADDS}

- For the first time, a study reports a very large clustering effect of self-harm in prisoners (ICC $=0.15$ ).

- The longitudinal observation provides an opportunity to identify risk factors associated with prisoners to be targeted by more intensive preventive programmes. Older age, a previous self-harm incident of moderate or high lethality for males, and a life sentence or more than five self-harm incidents in the previous year for females, are significantly associated with completed suicide among prisoners who self-harmed.

- Self-harm repetition rates, stratified by gender, offer valuable information for health professionals who work in prison. Female prisoners who self-harm will have an average of eight self-harm incidents per year and therefore require increased preventive efforts.

\section{LIMITATIONS}

- The risk factors that have been identified as associated with suicide have low predictive power and are not readily translatable into the clinical practice. Clinicians need screening tools with increased specificity in order to be able to identify those patients who are likely not to complete suicide.

- No information was available about completed suicides after release from jail/prison, which is a critical time with increased risk. ${ }^{4}$ The reported suicide rates only refer to suicide in prison and are therefore likely to underestimate the number of prisoners who self-harm in jail and subsequently die by suicide.

\section{WHAT NEXT IN RESEARCH?}

It is necessary to identify more risk factors that might be associated with suicide with higher specificity, such as personality characteristics and psychiatric diagnoses. This can be achieved through prospective cohort studies that specifically evaluate a large number of risk and protective factors at baseline and observe suicides after long-term follow-up. An alternative but powerful approach is to look at near-lethal deaths (rather than completed suicides); it has the advantage of not needing such large numbers. ${ }^{5}$ Such studies would require a major effort in terms of resources to be allocated but represent the only means for the development of tools that would be useful to clinicians who work with suicidal inmates. Such prospective studies should also consider biological predictors of suicide, such as genetic vulnerability factors. ${ }^{6}$

\section{DO THESE RESULTS CHANGE YOUR PRACTICES AND WHY?}

The results of the study do affect clinical practice in prison. Specifically, the reported large clustering effects suggest that any intervention to prevent self-harm should involve not only the individual prisoner but should target the whole population of a prison where self-harm occurs. Moreover, the high rate of repetitions reported for females (8 times per year) indicate that female prisoners who commit acts of self-harm require special attention and should be targeted by specific preventive programmes, starting from the first act of self-harm. The finding that female prisoners with more than five episodes of self-harm are at increased risk of suicide is especially important, as it represents a clear indication that repeated self-harm should not be considered manipulative or underestimated. On the contrary, it should be taken very seriously and requires firm preventive action.

Competing interests None.

doi:10.1136/eb-2014-101895

\section{REFERENCES}

1. Hayes LM. National study of jail suicide: 20 years later. J Correct Health Care 2012;18:233-45.

2. Carli V, Mandelli L, Postuvan V, et al. Self-harm in prisoners. CNS Spectr 2011;16:75-81.

3. Fazel S, Cartwright J, Norman-Nott A, et al. Suicide in prisoners: a systematic review of risk factors. J Clin Psychiatry 2008;69:1721-31.

4. Zlodre J, Fazel S. All-cause and external mortality in released prisoners: systematic review and meta-analysis. Am J Public Health 2012;102:67-75.

5. Rivlin A, Fazel S, Marzano L, et al. Studying survivors of near-lethal suicide attempts as a proxy for completed suicide in prisons. Forensic Sci Int 2012;220:19-26.

6. Thorell LH, Wolfersdorf M, Straub R, et al. Electrodermal hyporeactivity as a trait marker for suicidal propensity in uni- and bipolar depression. J Psychiatr Res 2013;47:1925-31.

ABSTRACT FROM: Hawton K, Linsell L, Adeniji T, et al. Self-harm in prisons in England and Wales: an epidemiological study of prevalence, risk factors, clustering, and subsequent suicide. Lancet 2014;383:1147-54.

Population Prisoners aged 15 years and over in prisons in England and Wales. Setting All prisons in England and Wales; January 2004 to December 2009.

\section{OUTCOMES}

Prevalence of self-harm Six to seven per cent of prisoners in England and Wales self-harmed over the 5-year study period. A total of 139195 self-harm incidents involving 26510 prisoners were recorded. The prevalence rate ranged 200-249 incidents per 1000 prisoners over the 5 years. Prevalence rates were consistently higher in females than males: an estimated 5-6\% of male prisoners self-harmed, compared with $20-24 \%$ of female prisoners. Cutting or scratching was the most common method of self-harm in males and females ( $65 \%$ and $51 \%$ of incidents, respectively).

Risk factors for self-harm In male and female prisoners self-harm was associated with younger age (less than 20 years), being of white ethnic origin, prison type (high security prison in males; mixed local prison in females), and being either unsentenced or given a life sentence. Females were also more likely to selfharm if they had previously committed a violent offence against an individual.

Suicide rates Among the prisoners who self-harmed at any time between 2004 and 2009, there were 109 subsequent suicides. Suicide rates were 450/100 000 in males and 259/100 000 in females. Comparatively, the rate of suicide in prisoners who had not self-harmed was 79/100 000 in males and 98/100 000 in females. There was no significant difference in the overall proportion of suicides between male and female prisoners ( $R R=1.74,95 \%$ CI 0.99 to 3.04 ). In men who had selfharmed, being aged 30-49 and a previous self-harm incident of moderate or high lethality were significant risk factors for suicide. In women who had self-harmed, serving a life sentence and a history of more than five self-harm incidents per year were significant risk factors for suicide. 